\title{
Poroelasticity of the Pre-salt Reservoir Rocks
}

Guilherme Fernandes Vasquez, Marcio José Morschbacher, Julio Cesar Ramos Justen, Marcos Pozzato Figueiredo, and Douglas Luiz Pinto de Lacerda. Petrobras.

Copyright 2019, SBGf - Sociedade Brasileira de Geofísica

This paper was prepared for presentation during the $16^{\text {th }}$ International Congress of the Brazilian Geophysical Society held in Rio de Janeiro, Brazil, 19-22 August 2019.

Contents of this paper were reviewed by the Technical Committee of the $16^{\text {th }}$ International Congress of the Brazilian Geophysical Society and do not necessarily represent any position of the SBGf, its officers or members. Electronic reproduction or storage of any part the Brazilian Geophysical Society is prohibited.

\section{Abstract}

The theory of Linear Poroelasticity is important in groundwater problems, civil engineering, as well as reservoir and geotechnical engineering, providing accurate results for several applications. Geophysics takes benefit from poroelastic properties as well, especially on rock physics related investigations. Gassmann's equations and Biot's theory, intimately related to poroelasticity, are widely applied on time-lapse feasibility and interpretation studies. Moreover, based on this theory, the derivation of important parameters for geomechanics from geophysical measurements is straightforward. These derivations may have the additional advantage to give high sampling compared to laboratory core testing, if well logs are used in the calculations, for instance. However, since the Brazilian pre-salt reservoirs comprises so complex and heterogeneous carbonate rocks, the applicability of poroelasticity for such rocks is often arguable. We estimate some poroelastic properties of pre-salt carbonates through laboratory measurements. The results are in good agreement with those derived from geophysical measurements. Furthermore, we tested the BiotGassmann model, measuring dry and saturated rock velocities in the laboratory. Our investigations so far endorses the applicability of the theory of poroelasticity to pre-salt carbonate rocks.

\section{Introduction}

Various geophysical and geomechanical applications assumes that rocks are elastic, homogeneous and isotropic media. This convenient and useful approximation allows simplifications in the formalism, the involved calculations, and provides results with negligible errors for several purposes. Nevertheless, rocks are far from being elastic materials. Inasmuch that rocks are porous media, or solid skeleton, with pores partially or fully saturated with fluids, it is possible to figure out the sophistication to consider the rocks as poroelastic materials.

Two basic phenomena are associated with the poroelastic behavior. First, the solid-fluid coupling, when a change in applied stress causes a change in fluid pressure or in the mass of fluid contained on the pores. Secondly, the fluidsolid coupling, when a change in fluid pressure or in the mass of fluid contained in the pores produces a change on rock or porous media total volume. (Wang, 2000)

Nowadays, reservoir engineers must consider the geomechanical effects associated to production (and injection) that may represent serious risks to the integrity of petroleum accumulations, installations and even the environment, as subsidence and fault reactivation, for instance. On the other hand, geophysicists applies poroelasticity results in the simulation and interpretation of the seismic response variations associated with fluid saturation changes, for example.

Although the stresses and strains involved in the propagation of seismic waves in rocks are several orders of magnitude lower compared to those associated with production, the Poroelasticity Theory represents a common language among geophysics and engineering.

The required parameters for geomechanical modeling can be inferred from well log data, laboratory experiments or even seismic data. However, it must be considered that parameters derived from geophysical methods may not be adequate to geomechanical models. Generally, they are not. One of the reasons is the difference in magnitude of the variations in strain and stresses involved. Another reason is that, in spite of its elegance, consistency and robustness, Poroelasticity is still an approximation to describe rock behavior.

Poroelastic properties of reservoir rocks will be discussed herein with a geophysical bias. Results obtained from investigations aiming to estimate poroelastic parameters from geophysical measurements will be presented. Furthermore, we compare these estimates of poroelastic parameters with derivations via petrophysical and engineering analysis, in order to verify the applicability of poroelasticity theory for pre-salt carbonates.

\section{Poroelastic Coefficients in Rock Physics}

Two important poroelastic coefficients are considered herein: Biot's coefficient and Skempton's coefficient.

The Biot's coefficient (Biot and Willis, 1947) is a measure of the difference between the drained bulk modulus of a rock, when the fluid is free to flow in and out of the pores, $K_{d}$ (ideally identical to the dry rock bulk modulus), and the grain's or solid matrix bulk modulus, $K_{m}$ :

$$
\alpha=1-\frac{K_{d}}{K_{m}}
$$

This coefficient is very important in geomechanics because it corresponds to the effective stress coefficient for bulk compressions of rocks. It means that, if a rock is subjected to a confining pressure $P_{c}$ and the pore fluid to a pore 
pressure $P_{p}$, the value of the bulk modulus of this rock will be equal to that modulus for the same rock subjected to an effective stress $P_{e}=P_{c}-\alpha P_{p}$. Nur and Byerlee (1979) had shown this property in an elegant way, assuming only that elastic grains compose the rock matrix.

In geophysics, Biot's coefficient gives a measure of the sensitivity of rock's elastic properties to fluid replacement, especially for compressional-wave velocity and associated properties, as will be seen later.

Some authors believe that the Biot's coefficient corresponds to the effective pressure coefficient for elasticwave velocities. It seems to be not true, since it is refuted by several theoretical (e.g.: Berrymann, 1993; Gurevich, 2004; Müller and Sahay, 2016) and experimental (e.g.: Christensen and Wang, 1985, Prasad and Manghnani, 1997, Xu et al., 2006, Vasquez et al., 2010) investigations.

Skempton (1954) defined two poroelastic coefficients, one of which is known as Skempton's coefficient $B$. It gives a measure of pore pressure variation on a rock associated with a change in confining stress under undrained condition (without allowing the fluid to get out of the pore volume):

$$
B=\frac{\Delta P_{p}}{\Delta P_{c}}
$$

According to the theory of linear poroelasticity, Rosa (2010) shows that there is a relation between these two coefficients:

$$
\frac{1}{B}=1+\frac{\phi K_{d}}{\alpha}\left(\frac{1}{K_{f}}-\frac{1}{K_{m}}\right)
$$

where $\phi$ is the rock porosity and $K_{f}$ the pore fluid incompressibility.

The link between poroelasticity and geophysics can be established through the compressional- and shear-wave propagation velocities. Considering the isotropic case:

$$
\begin{gathered}
V_{P}=\sqrt{\frac{K+\frac{4}{3} \mu}{\rho}} \\
V_{S}=\sqrt{\frac{\mu}{\rho}}
\end{gathered}
$$

Therefore, from the geophysics point of view, if the velocities and the density are known, it is possible to calculate the elastic, or poroelastic, moduli (bulk modulus, $K$, and shear modulus, $\mu$ ). Both moduli and density depends on the fluid saturating the pore space. In the case of a dry rock, with no fluid in pores, that is an approximate condition to some laboratory situations or to shallow soil, the density can be estimated from porosity $\phi$ and solid matrix density $\rho_{m}$,

$$
\rho_{d}=(1-\phi) \rho_{m}
$$

On geophysics the dry rock bulk modulus is equal to the drained bulk modulus from geomechanics, $K_{d}$. It comes from the fact that, in drained geomechanic experiments, with the fluid free to get in or out the pores at very low strain rates, the fluid offers no resistance to the frame deformation.

On the other hand, as an elastic-wave travels through a rock, although it causes extremely tiny strains $\left(<10^{-6}\right)$, these happens at higher frequencies, greater than one cycle per second, being typically on the range 10 up to 40 cycles per second on seismic methods, and around $10^{6}$ cycles per second on laboratory experiments. Under these conditions the rock may be consider as undrained, that is, there is not enough time for the fluid to get out and return to the porous space as the wave compress or elongate the rock.

Gassmann (1951) derived the elastic moduli of undrained rocks. For the bulk modulus:

$$
K=K_{d}+\frac{\left(1-\frac{K_{d}}{K_{m}}\right)^{2}}{\frac{\phi}{K_{f}}+\frac{1-\phi}{K_{m}}-\frac{K_{d}}{K_{m}^{2}}}
$$

Note that the numerator on the second term on the right hand side on equation (7) corresponds to the Biot's coefficient squared. It may be observed that, as this term get closer to 1 , the rock's sensitivity to fluid substitution becomes larger.

Many geophysicists considers that the rock are more sensitive to fluid changes as $K_{d}$ gets smaller. Although it is partially true, in fact, the determining factor is the Biot's coefficient. As an example, if we could have a quartz rich sandstone and a clean carbonate with similar porosities and similar dry bulk modulus, the limestone would be more sensitive to fluid substitution because the grain modulus is near $37 \mathrm{GPa}$ for quartz, whereas for the calcite the modulus is around $75 \mathrm{GPa}$. The reader is invited to make some simulations and verify these statements.

According to poroelasticity and the estimates from Gassmann model, the shear modulus remains unchanged with saturation. It is somewhat intuitive, since this model was conceived for non-viscous fluids, which does not resists to shearing.

The rock density, when saturated with a fluid with density $\rho_{f}$, will be:

$$
\rho=(1-\phi) \rho_{m}+\phi \rho_{f}
$$

\section{Inference or Estimation Methods}

The methods used to estimate the poroelastic coefficients of the rocks will be described shortly.

Compressional- and shear-wave velocities were measured by the ultrasonic pulse transmission technique with an AutoLab 1000 system (made by New England Research). The experimental system allows the measurement of one compressional and two shear waves, with mutually orthogonal polarizations. For all these wave modes, the propagation direction is along the longitudinal or symmetry axis of the core plugs, which has cylindrical shape. In order to derive the elastic moduli under the isotropy assumption, the average value between the two shear-wave measurements was assigned to the shear-wave velocity. 
Porosity, matrix density and permeability were measured with an helium porosimeter-permeameter, and rock composition was estimated by X-ray diffractometry (XRD).

Skempton's coefficient estimation was made on the velocity measurement system. For selected confining and pore pressure values, we imposed the undrained condition, so that the fluid volume on pores (and tubes) was constant. Then confining pressure variations were imposed and the associated pore pressure changes were recorded. The confining pressure oscillations were made according to triangular periodical functions, with quite low frequencies, as exemplified on Figure 1.

For the Biot's coefficient measurement, it is necessary to measure the dry rock bulk modulus and the solid matrix bulk modulus as well. It requires the measurement of rock (and matrix) deformations in response to applied stresses. Thus, the samples were first involved with a copper jacket and instrumented with strain gages in order to monitor the longitudinal and radial deformations. The strain gages are electronic resistors which resistance varies with deformations. Four Vishay model EA-09-500BH-120/LE, with $120 \mathrm{Ohm}$ resistance were used, two for longitudinal and two for radial deformation measurements. Each strain gage must be connected in a Weathstone bridge, which is an electric circuit for accurate resistance measurements. The volumetric strain can be calculated with the axial and radian strains, respectively $\varepsilon_{a}$ and $\varepsilon_{r}$, according to:

$$
\varepsilon_{V}=\frac{\Delta V_{0}}{V_{0}}=\frac{1}{3}\left(\varepsilon_{a}+2 \varepsilon_{r}\right)
$$

The bulk modulus measurements was made for selected confining and pore pressure conditions, forcing the confining pressure variation with the pore pressure maintained constant. In order to measure the solid grain bulk modulus it is necessary to have equal pore and confining pressure (and fluid), and impose identical variations on both, such that the total strain of the rock will be due only to change in the solid matrix volume.

The Biot's coefficient derived from the stress-strains experiments may be named as static, or quasi-static, as the stress and the strain variations occurs at very low frequencies, approaching zero when compared to those related with seismic waves. We named dynamic Biot's coefficient those ones estimated from the rock bulk modulus derived from elastic-wave velocity measurements.

\section{Results}

For the sake of space, we illustrate only few examples of estimates and verification of the validity of poroelasticity theory.

We exemplify the estimate of the Skempton's coefficient with the case of sample \#546V, collected at 5764.2 meters depth from one oil well of a pre-salt oil field. Its basic petrophysical properties are listed in Table 1. Initially, the compressional and shear-wave velocities were measured in the dry sample. Subsequently the sample was saturated with ethanol, which does not chemically interact with the carbonate matrix and presents an incompressibility of the order of $1.18 \mathrm{GPa}$ under ambient conditions, similar to some in situ oil-water mixtures. Compositional analysis shows that this rock is formed almost entirely by Calcite, having $6 \%$ of Dolomite.

Figure 1 illustrates the confining pressure variations and associated pore pressure changes observed for a given series of cycles. Several confining pressure cycling series were made with different amplitudes and frequency of confining pressure variation. Applying Equation (2) we arrive at Skempton's coefficient values between 0.16 and 0.17 , according to the series of cycles used. In Figure 2 is illustrated the example of a cross plot with the confining pressure variations and consequent pore pressure changes for a series of cycles on which Skempton's coefficient estimate was 0.16 .

According to the theory of poroelasticity, starting from dry rock velocity values and using Equation (3), we estimate the value of 0.18 for the Skempton's coefficient of the sample saturated with ethanol, which agrees with the direct experimental estimates. That is, for this particular rock, the Skempton coefficient calculated from geophysical properties agrees with the one obtained from direct measurement.

One of the problems in the experimental determination of the Skempton's coefficient is related to the volume of fluid in the pressure lines. Ideally, it must be much smaller than the volume of fluid in the rock. We planned some adaptations in the equipment to improve repeatability and accuracy in these tests.

We also observed that for cycles of confining pressure with amplitudes greater than $5 \mathrm{MPa}$ the phenomenon deviates from linearity. The graph on Figure 2, for example, exhibits a slightly non-linear response.

Table 1 - Basic petrophysical properties (porosity $\phi$, permeability $\kappa$ and solid density $\rho_{m}$ ) of sample \#546V.

\begin{tabular}{ccc}
\hline$\phi(\%)$ & $\kappa(\mathrm{mD})$ & $\rho \mathrm{m}\left(\mathrm{g} / \mathrm{cm}^{3}\right)$ \\
\hline 14.7 & 5.36 & 2.75 \\
\hline
\end{tabular}

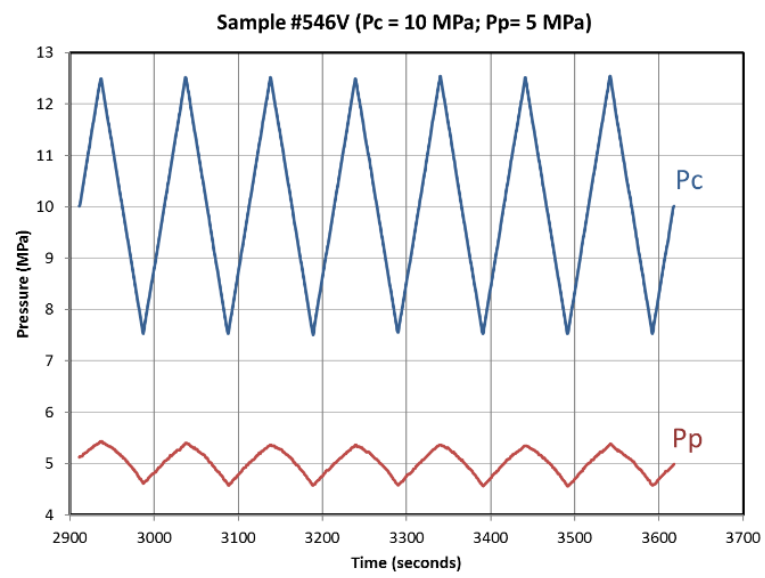

Figure 1 - Series of oscillations of the confining pressure and consequent pore pressure variations in sample \#546V. 


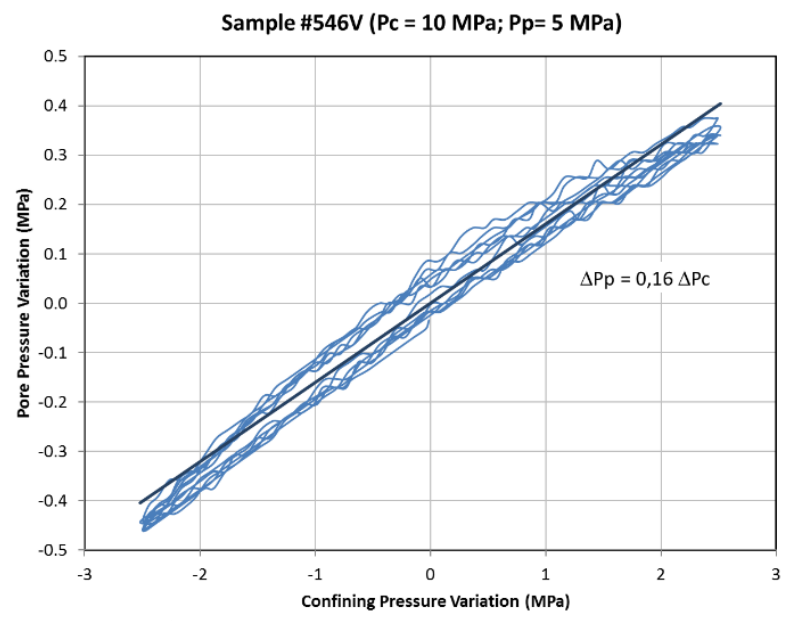

Figure 2 - Cross plot of variation of the confining pressure and pore pressure variations in sample \#546V.

On the experiments to estimate the Biot coefficient, it is necessary to perform the measurement of the dry rock bulk modulus and the grains bulk modulus, which requires the instrumentation of the sample with strain sensors. In our case, we use resistive strain gages (or strain gauges). Figure 3 illustrates a photograph of an instrumented sample for deformation measurements.

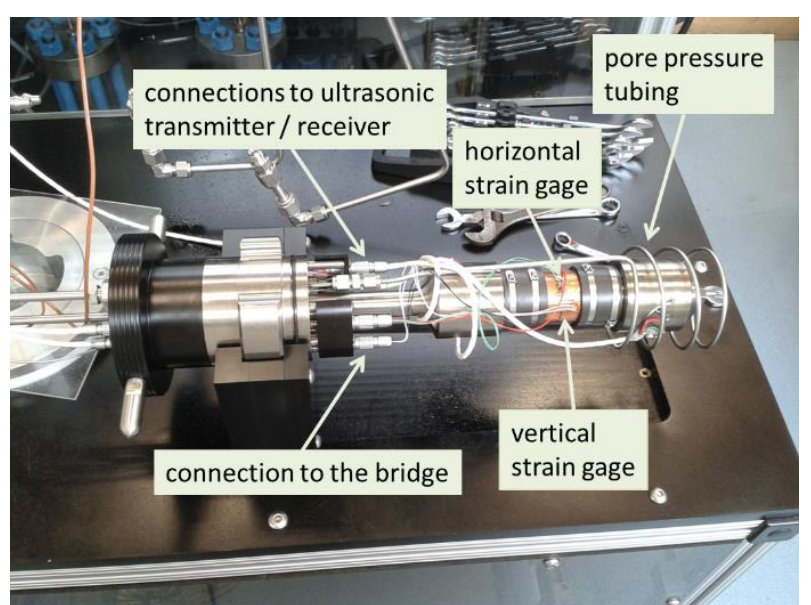

Figure 3 - Photograph of an instrumented sample for volumetric stress-strain measurements.

For the measurement of the grains or solid fraction bulk modulus, the pore pressure must be maintained equal to the confining pressure and both must be varied by the same amounts. Under these conditions, the volume variation of the rock will be equal to the volume variation of the grains. Evidently, in this case the deformations are much smaller, making the estimates even more difficult.

Herein we show examples of results of experiments performed with a rock sample from Barra Velha Formation collected at 5011.15 meters depth, \#687H. Table 2 lists its basic petrophysical properties and Table 3 the mineralogical composition according to XRD analyzes.

Table 2 - Basic petrophysical properties (porosity $\phi$, permeability $\kappa$ and solid density $\rho m$ ) of sample \#687H.

\begin{tabular}{ccc}
\hline$\phi(\%)$ & $\kappa(\mathrm{mD})$ & $\rho \mathrm{m}\left(\mathrm{g} / \mathrm{cm}^{3}\right)$ \\
\hline 14.7 & 5.36 & 2.75 \\
\hline
\end{tabular}

Table 3 - mineralogical composition (\%) obtained by $X$-ray diffraction in sample \#687H.

\begin{tabular}{ccccc}
\hline Dolomite & Quartz & Calcite & Dawsonite & Fluorite \\
\hline 19 & 18 & 57 & 5 & 1 \\
\hline
\end{tabular}

The graph on Figure 4 shows an example of confining pressure variation as a function of the resulting volumetric strain of the sample, for determination of the drained rock bulk modulus. In order to construct this graph, the confining pressure was initially set at $50 \mathrm{MPa}$ and the pore pressure at $10 \mathrm{MPa}$ (approximately). Then the pore pressure was maintained constant, while compression and dilatation cycles were imposed, increasing the confining pressure to 52.5 $\mathrm{MPa}$ and decreasing to $47.5 \mathrm{MPa}$ several times in cycles in the form of a triangular wave. From this specific experiment, the bulk modulus of the rock was estimated as $25 \mathrm{GPa}$.

The comparison of the rock bulk modulus obtained through stress-strain (static) tests with the one derived from dry rock velocity measurements (dynamic) for various differential pressures (difference between confining pressure and pore pressure) shows a very good agreement, with differences at most on the order of $1 \mathrm{GPa}$. This is an evidence of the validity of poroelastic theory for this rock, since the modulus derived from geophysics agrees with the one measured by quasi-static experiments.

The cross plot on Figure 5 shows an example of confining (and pore) pressure variation as a function of the resulting volumetric strain of the sample, for estimate the grain bulk modulus. To construct this graph, the confining and pore pressures were initially fixed at $50 \mathrm{MPa}$ and then compression and relaxation cycles were imposed, increasing both confining and pore pressure up to $54 \mathrm{MPa}$ and decreasing to $46 \mathrm{MPa}$ several times, in cycles in the form of a triangular wave. In this case, the grain bulk modulus was estimated as $74 \mathrm{GPa}$. Note that, in addition to calcite, this rock contains quartz (whose bulk modulus is lower than the one from calcite) and dolomite (bulk modulus higher than the one from calcite). Curiously, the measured bulk modulus for the solid mixture that compose the rock matrix is approximately to the calcite bulk modulus.

It is interesting to note that the strains involved in the graph from Figure 5 are much smaller than the strains in the graph from Figure 4, since it represents the deformation of the grains, much more rigid than the rock as a whole. Consequently, the graph shows that the data are contaminated by "noise", associated with the greater uncertainties and fluctuations. 
Figure 6 shows the static (or quasi-static) Biot's coefficient obtained from the measurements of the rock bulk modulus and the solid fraction modulus, calculated according to equation (1). In the same graph, the Biot's coefficient, also estimated with equation (1), but using the bulk modulus of the rock derived from the compressional and shear-wave velocities measured in the dry rock, is represented. There is a very good agreement between the behaviors and among the values of the coefficient estimated by the two methods.

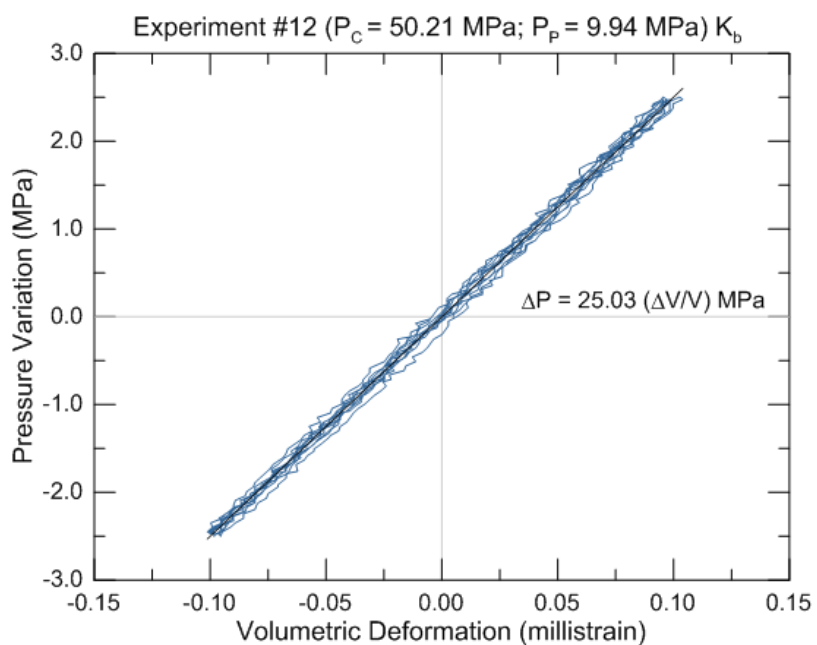

Figure 4 - Example of cross plot of confining pressure variation as a function of volumetric strain in sample $\# 687 \mathrm{H}$, used to estimate the drained bulk modulus. In such experiments the pore pressure is maintained constant.

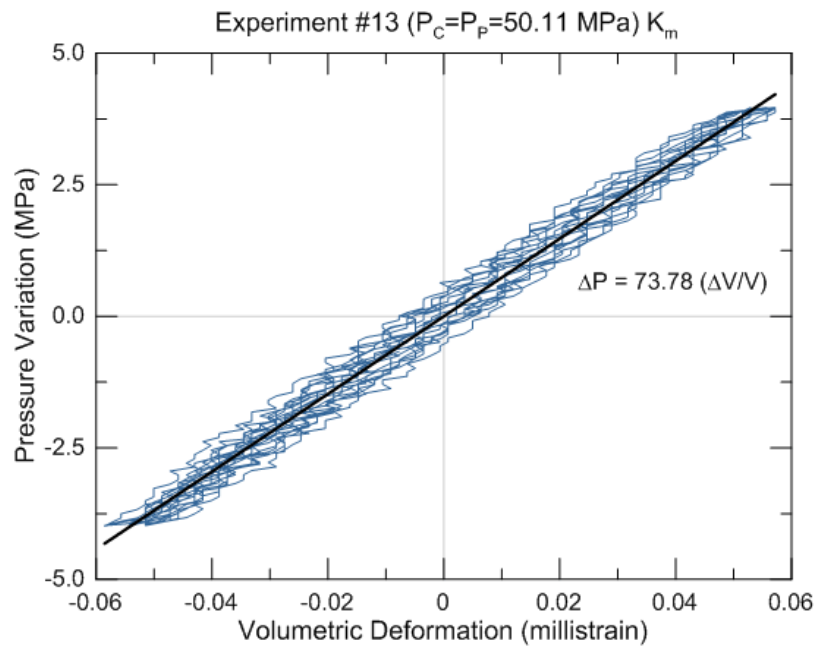

Figure 5 - Cross plot of confining pressure variation as a function of the volumetric deformation for sample \#687H, to estimate the grain bulk modulus. In this experiment, the pore pressure is identical to the confining pressure. Note that the volumetric strains in this case are much smaller than those shown in Figure 4.

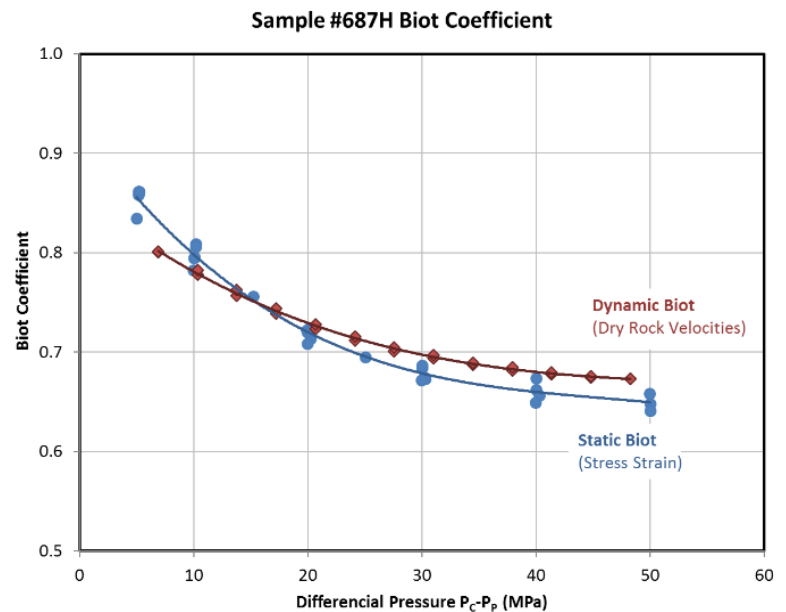

Figure 6 - Estimated Biot's coefficient for sample \#687H estimated via stress strain tests (blue discs) and via dry rock velocity measurements, using the measured grain bulk modulus (red diamonds).

Finally, as an example of the verification of the validity of the Gassmann model, Figure 7 illustrates the values of the bulk modulus of the rock sample \#546V, calculated from dry rock velocities, and from the velocities of the rock saturated with a brine with $30000 \mathrm{ppm} \mathrm{NaCl}$ and with ethanol, along with the predictions estimated by Equation (7). It is important to note that, for these calculations, the grain modulus was estimated from the composition provided by the XRD analysis.

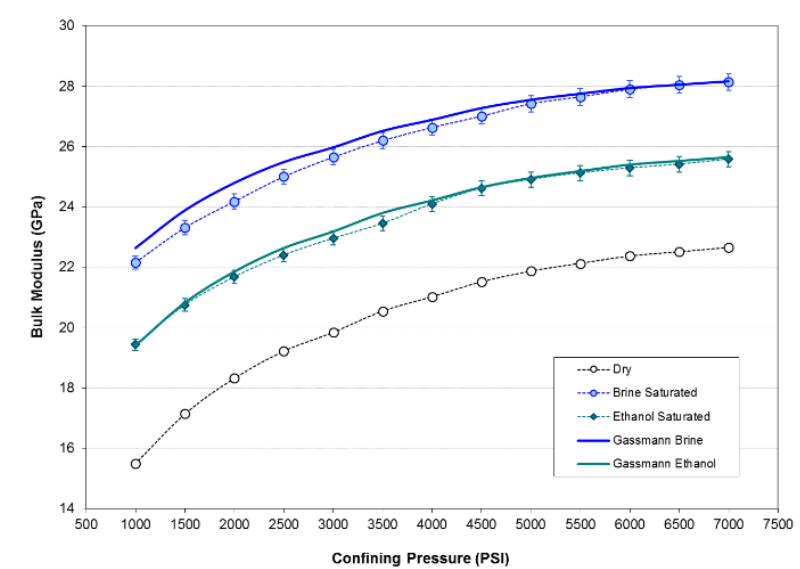

Figure 7 - Bulk modulus of sample \#546V, dry and saturated with brine and ethanol (black, blue and green dashed curves with data points, respectively), superimposed with the predictions according to the BiotGassmann model (continuous curves). 


\section{Conclusions}

We have shown examples of estimates of poroelastic properties of pre-salt reservoir carbonate rocks from direct measurement as well as from geophysical properties. The obtained agreement between geophysical estimations and direct measurement can be interpreted as an experimental verification of the validity of the linear poroelasticity theory for these rocks. It is an evidence that this theory can be used to describe the mechanical and acoustic behavior of the pre-salt reservoir rocks. These observations has at least two very important implications in exploration and reservoir geophysics. First, it suggests that we can use poroelastic properties derived from geophysical measurements for geomechanical applications. Secondly, it suggests that we can apply the Gassmann model to simulate fluid substitution on pre-salt carbonate rocks.

\section{Acknowledgments}

We thank very much Dr. Marcia Filardi for review and useful suggestions and Petrobras for support and permission to publish these results. We are also grateful to Alvaro Arouca, José Lira and Vinicius Machado for their backing on this research and Andre Romanelli Rosa for the encouraging enquiring the petroelastic properties of rocks in the lab.

\section{References}

Berryman, J. G., 1993. Effective stress rules for pore-fluid transport in rocks containing two minerals. International Journal of Rock Mechanics, 30, 1165-1168.

Biot, M. A. and Willis, D. G., 1957. The elastic coefficients of the theory of consolidation. ASME Journal of Applied Mechanics, 24, 594-601.

Christensen, N. I., and Wang, H. F., 1985. The influence of pore pressure and confining pressure on dynamic elastic properties of Berea sandstone. Geophysics, 50, 207-213.

Gassmann, F., 1951. On Elasticity of Porous Media. Translated in: Classics of Elastic Wave Theory. Geophysics Reprint Series No. 24. Pelissier, M.A., Hoeber, H., de Coevering, N. and Jones, I.F. (eds.), 389-407. Society of Exploration Geophysicists, Tulsa, 2007.

Gurevich, B., 2004. A simple derivation of the effective stress coefficient for seismic velocities in porous rocks. Geophysics, 69, 393-397.

Müller, T.M. and Sahay, P.N. 2016. Biot coefficient is distinct from effective pressure coefficient. Geophysics, 81, L27-L33.

Nur, A. and Byerlee, J. D., 1971. An exact effective stress law for elastic deformation of rocks with fluids. Journal of Geophysical Research, 76, 6414-6419.

Prasad, M., and Manghnani, M., 1997. Effects of pore and differential pressure on compression wave velocity and quality factor in Berea and Michigan sandstones. Geophysics, 62, 1163-1176.
Rosa, A.L.R. 2010. Análise do Sinal Sísmico. Sociedade Brasileira de Geofísica, Rio de Janeiro. 668 p.

Skempton, A.W. 1954. The Pore-Pressure Coefficients A and B. Géotechnique, 4, 143-147.

Vasquez, G., Vargas Jr., E., Justen, J. and Morschbacher, M. 2010. How Effective Is the Effective Stress Coefficient - An Experimental Study on Brazilian Rocks. 72nd EAGE Conference and Exhibition incorporating SPE EUROPEC 2010. June 14-17. Barcelona, Spain.

Wang, H.F. 2000. Theory of Linear Poroelasticity with Applications to Geomechanics and Hydrogeology. Princeton University Press. New Jersey. 287 p.

Xu, X., Hofmann, R., Batzle, M., and Tshering, T., 2006. Influence of pore pressure on velocity in low-porosity sandstone: Implications for time-lapse feasibility and porepressure study. Geophysical Prospecting, 54, 565-573. 